\title{
New Targets for Immune Modulation in Asthma
}

\section{Willem van de Veen, PhD \\ Mübeccel Akdis, MD, PhD*}

\author{
Address \\ * Swiss Institute of Allergy and Asthma Research, University of Zürich, \\ Oberestrasse 22, CH-7270, Davos Platz, Switzerland \\ Email: akdism@siaf.uzh.ch
}

Published online: 30 January 2014

(C) Springer International Publishing AG 2014

Keywords Asthma - Biologicals - Allergen specific immunotherapy - Allergen SIT - Endotypes ·

Immunomodulation · IgE · IgG4 · Eosinophilia · Treg • Omalizumab · Breg

\section{Opinion statement}

Curative treatment for asthma poses a challenge. Standard asthma therapy is primarily based on immunosuppressive and bronchodilator drugs, which confer shortterm symptom relief but do not cure the disease. Approximately 5-10\% of asthma patients do not respond to conventional therapy. Currently, allergen-specific immunotherapy (SIT) is the only available curative treatment for allergic asthma patients, and is most effective in monosensitized individuals. Toll-like receptor (TLR) 4 and TLR9 agonists can be used as an adjuvant to improve the efficacy of allergen-SIT. Anti-IgE therapy is currently used with varying success rates for the treatment of allergic asthma. Appropriate diagnosis and recognition of the etiology of underlying disease symptoms is essential for the success of novel immunomodulatory treatments. Disease classification based on endotypes will facilitate the appropriate choice for therapy, as endotypes describe subtypes of asthma, which are defined by distinct pathological mechanisms. Novel immunomodulatory therapies are targeting specific cytokines or cell-surface receptors and interfere with these pathological mechanisms, thereby altering the course of the disease. A range of novel immunomodulatory drugs is currently under development for the treatment of asthma. These drugs specifically target key molecules that play a role in the pathways involved in the pathogenesis of asthma, and include biologicals that primarily target cytokines and cytokine receptors, as well as small molecules that target chemokine receptors and TLRs. The safety and efficacy of many of these novel drugs still remain to be determined.

\section{Introduction}

Asthma is a chronic inflammatory lung disease that affects an estimated 300 million individuals worldwide
[1]. The main characteristics of asthma are airway inflammation, bronchial hyperresponsiveness, and reversible 
airflow obstruction. Asthma patients may exhibit several or all of these characteristics, and it is clear that asthma cannot be considered as a single disease, but rather as an umbrella term that describes a variety of lung diseases with different underlying etiologies.

Currently, asthma is classified according to the phenotype of the disease. Due to the high level of heterogeneity of the disease, it is challenging to classify asthma into well-defined phenotypes. Initially, asthma was separated into two distinct phenotypes: extrinsic (also referred to as allergic) and intrinsic (also referred to as non-allergic) asthma. Extrinsic asthma exacerbates following respiratory exposure to known allergens, while intrinsic asthma shows no clinical or serological evidence of IgE-mediated reactions to these allergens (indicating a different mechanism of disease or failure to identify the relevant allergen) [2]. Identification of the type of granulocyte infiltration in induced sputum has been applied to classify asthma phenotypes and has proven its value for predicting treatment responses as it provides information on the mechanism of the disease pathogenesis. Based on the proportions of neutrophils and eosinophils in sputum, the following phenotypes can be identified: neutrophilic asthma (elevated sputum neutrophils), eosinophilic asthma (elevated sputum eosinophils), mixed granulocytic asthma (elevated eosinophils and neutrophils), and paucigranulocytic asthma (normal levels of eosinophils and neutrophils). Recent studies have applied advanced mathematical tools such as cluster analysis to more specifically identify asthma phenotypes $[3,4]$.

Despite the fact that these phenotypes are often clinically relevant, they frequently fail to give insight into the pathogenesis underlying the observed symptoms. Recently, another classification system for asthma was proposed based on so-called endotypes, which describe subtypes of a condition and are defined by a distinct functional or pathophysiological mechanism $[5 \bullet, 6]$. This novel classification is promising as it aims to describe distinct disease entities with a defining etiology, and is expected to facilitate better therapy that specifically targets the cause of the disease. So far, no consensus has been reached on defining asthma endotypes, and confirmation of proposed endotypes is still required. Recently, five asthma endotypes with associated characteristics were proposed: (1) allergic asthma [eosinophil infiltration, $\mathrm{T}$ helper (Th) 2-mediated inflammation and responsiveness to steroids, allergen-SIT, anti-interleukin (IL)-5 and anti-IL-4/-IL-13 therapy]; (2) intrinsic asthma (eosinophil infiltration/neutrophil infiltration, association with autoantibodies and superantigens); (3) neutrophilic asthma [innate immune response activation, abnormal histone deacetylase 2 recruitment, neutrophil infiltration, steroid resistance and responsiveness to antibiotics, antitumor necrosis factor (TNF)- $\alpha$ and histone deacetylase regulators]; (4) aspirin-intolerant asthma (eosinophil infiltration, altered eicosanoid metabolism, responsive to steroids and leukotriene receptor antagonists); and (5) extensive remodeling asthma (lack of inflammation, airway smooth muscle abnormalities, steroid resistance and responsiveness to therapies targeting airway smooth muscle, matrix metalloproteases, and angiogenesis) [2]. A certain endotype, for example allergic asthma, can have different disease phenotypes, such as eosinophilic asthma, exacerbation-prone asthma, or exercise-induced asthma. On the other hand, illustrating the difficulties resulting from the use of disease phenotypes, a given phenotype (e.g., eosinophilic asthma) can have several etiological backgrounds and might present in the context of a range of asthma endotypes, such as allergic asthma, aspirin-sensitive asthma, or intrinsic asthma.

Successful treatment critically depends on the correct identification of the etiology of the disease symptoms. Anti-inflammatory drugs frequently used to treat asthma include glucocorticoids, antihistamines, and leukotriene receptor antagonists. These therapies temporarily relieve disease symptoms but do not prevent the chronic course of asthma. Here we focus on different immunomodulatory strategies that act more specifically on the different pathways involved in the pathogenesis of asthma. Treatment options that are currently used in practice, as well as novel therapies that are still under development, are discussed.

\section{Treatment}

- The treatment options listed below are focused on novel immunomodulatory strategies for the treatment of asthma patients who do 
not respond to classical immunosuppressive therapies such as glucocorticoids, antihistamines and leukotriene receptor antagonists. Table 1 summarizes immunomodulatory therapies that have been approved for the treatment of asthma or are in clinical phase 2 or beyond.

- The prevalence of asthma has significantly increased during the past 50 years, especially in industrially developed nations. Since this timeframe is too short to allow major changes in the genetic makeup of the population, it is likely that lifestyle has a major influence on the development of asthma. The major lifestyle-associated factors that have been linked to the development of asthma are tobacco smoke exposure, obesity, and the microbiome.

- Tobacco smoke exposure is a risk factor for the development of asthma. Parental smoking has been linked to childhood asthma and wheezing, while active smoking is associated with adult-onset asthma $[7,8]$.

- Obesity has been associated with an increased risk for the development of asthma and prevention of obesity is likely to reduce its incidence. In most cases the onset of obesity precedes that of asthma, suggesting that obesity could promote asthma development. Several mechanisms have been proposed that might play a role in this process. Obesity-associated systemic inflammation leads to increased production of pro-inflammatory mediators such as TNF- $\alpha$ and IL-6 and eotaxin[9]. Obesity also leads to mechanical changes in lungs such as reduced functional reserve capacity [9].

- The role of the microbiome in the pathogenesis of allergic diseases and asthma is currently the subject of intensive research. The potential of altering the intestinal flora through the use of probiotics for the treatment of asthma remains unclear. Administration of probiotics has been associated with reduced IgE production but not with protection against asthma [10]. Interestingly, children who grow up on a farm have a reduced risk of developing allergies and asthma. Several factors, including intake of raw milk, contact with livestock, and contact with animal feed, have been linked with this protective effect [11].

- Drug development for the treatment of asthma has become increasingly focused on biological immunomodulators such as therapeutic antibodies, soluble receptors, and cytokines, as well as small molecules [12]. These novel drugs typically target a single molecule involved in the pathogenesis of asthma. This targeted approach is 


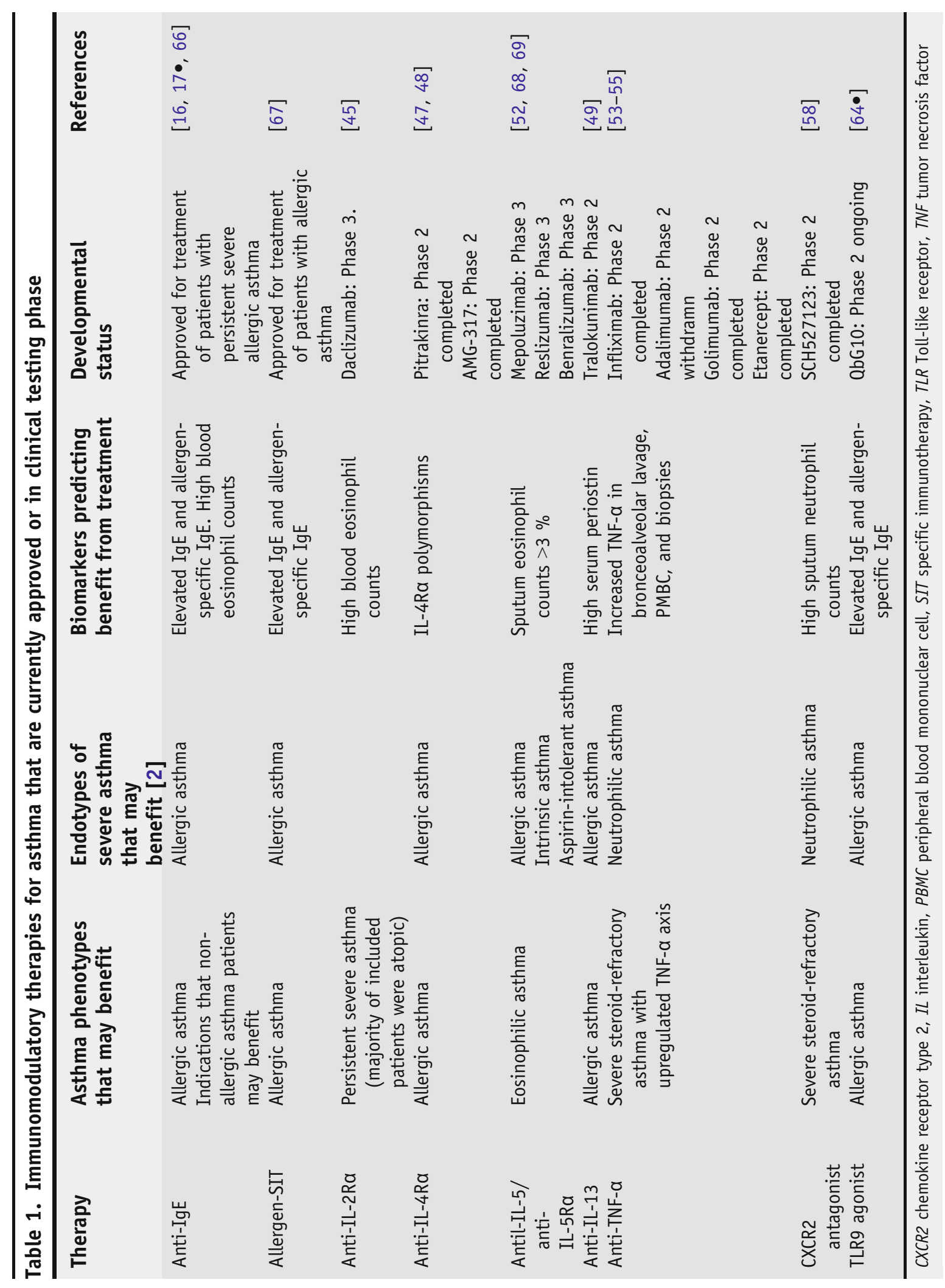


expected to result in the development of specific drugs that will benefit a certain group of asthma patients with a specific endotype. The success of the development of these novel drugs critically depends on correct patient stratification during the clinical testing phase.

\section{Biologicals}

Anti-IgE therapy (omalizumab)

- IgE is a key player in the pathogenesis of allergic asthma and was recognized early on as a potential therapeutic target. Anti-IgE therapy using the humanized anti-IgE antibody omalizumab (XOLAIR ${ }^{\circledR}$; Novartis) currently represents the only approved biological drug for the treatment of moderate to severe persistent allergic asthma that is not controlled by inhaled corticosteroids. Omalizumab binds to the Fc portion of unbound IgE and depletes free IgE. However, it cannot bind FceRI-bound $\mathrm{IgE}$, which prevents it from crosslinking FceRI and inducing subsequent mast cell or basophil degranulation. Interestingly, depletion of free IgE also leads to downregulation of FceRI expression on dendritic cells (DCs) and basophils, rendering these cells less sensitive to allergen exposure $[13,14]$. Omalizumab anti-IgE therapy leads to significant reductions in bronchial and nasal eosinophils and in bronchial $\mathrm{IgE}^{+}$mast cells as well as T and B cells. Omalizumab treatment leads to reduction of asthma exacerbations and symptoms in patients suffering from allergic rhinitis and asthma, allowing a reduction of the required maintenance dose of inhaled glucocorticoids $[15,16]$. A recent trial demonstrated that the response to omalizumab treatment differed according to patients' blood eosinophil counts at baseline. Patients with high initial eosinophil counts showed a $45 \%$ reduction in the incidence of exacerbations, while patients with low initial eosinophil counts showed no improvements [17•].

- The use of anti-IgE therapy in children has not been extensively studied. A recent clinical trial, which included inner-city children, adolescents, and young adults with persistent allergic asthma, demonstrated a $24.5 \%$ decrease in the frequency of reported asthma symptoms and $a>20 \%$ reduction in the number of patients who had one or more exacerbations [18 $\bullet$. These findings support efficacy of omalizumab treatment in children.

- Omalizumab is typically administered subcutaneously at 2- or 4week intervals at a dose of 150-375 mg (depending on body weight and baseline serum IgE level).

- Besides a previously observed hypersensitivity reaction to omalizumab, there are no specific contraindications to the use of omalizumab. No major drug interactions have been reported.

- Anaphylaxis was reported in three of 3,507 (0.1\%) allergic asthma patients in clinical trials. Furthermore, the incidence of malignancy 
among omalizumab-treated patients was higher $(0.5 \%)$ than that observed in the placebo group (0.2\%) [19]. The clinical data, however, do not suggest a causal relationship between omalizumab treatment and cancer as the onset of most of these tumors preceded the treatment [15]. Other reported side effects include rash at injection site, gastrointestinal complaints (nausea, diarrhea, abdominal pain), and bleeding (nosebleed, increased menstruation bleed).

- Omalizumab treatment is relatively costly. The annual costs vary per patient depending on frequency and dosage. Recent estimations by the British National Institute for Health and Care Excellence (NICE) on the annual cost of omalizumab therapy vary between $£ 1,665$ (for a $75 \mathrm{mg}$ dose every 4 weeks) and approximately $£ 26,640$ (for a $600 \mathrm{mg}$ dose every 2 weeks). The incremental cost-effectiveness ratio for adults was estimated to be around $£ 83,822$ per quality-adjusted life-year gained [16].

- Allergen-specific immunotherapy (SIT) is the only available treatment that can induce long-term improvement of clinical allergy symptoms. The earliest reports on the protective effects of allergenSIT date back to the beginning of the twentieth century, when Noon and Freeman reported clinical improvement in hay fever patients after applying subcutaneous injections of grass pollen extracts [20, 21]. The basic principle of allergen-SIT has not changed much since then. This principle comprises repeated administration of increasing doses of the causative allergen. Allergen-SIT is most frequently applied for the treatment of hymenoptera venom allergies and allergic rhinitis caused by grass or tree pollen, as well as house dust mites [22].

- Allergen-SIT acts on different levels of the immune system and induces changes on eosinophils, mast cells, basophils, T cells, B cells, and allergen-specific immunoglobulins.

- Many patients who receive allergen-SIT become tolerant to late-phase skin responses at early stages of the therapy. One of the events associated with this observation is that mast cell and basophil degranulation activity is reduced already after the first administration of allergen. The mechanism underlying this effect remains largely unknown. A role for histamine receptor (HR) 2 in the suppression of basophil degranulation has been suggested. A rapid upregulation of HR2 expression on basophils was observed in bee venom allergic patients receiving SIT. HR2 expression was strongly upregulated within $6 \mathrm{~h}$ after the initiation of SIT and suppressed FceRI-mediated basophil degranulation [23•].

- Induction of a tolerant state in peripheral T cells is another critical step for the success of allergen-SIT. Key cytokines that mediate peripheral tolerance are IL-10 and transforming growth factor (TGF)- $\beta$. 
Peripheral tolerance is characterized mainly by the induction of allergen-specific type 1 regulatory $(\operatorname{Tr} 1)$ cells, which produce IL-10 and TGF- $\beta$ [24, 25]. Interestingly, CD $4+C D 25+T$ regulatory (Treg) cells from atopic donors less efficiently suppress proliferation of effector $\mathrm{T}$ cells than Treg cells from healthy donors [26].

- Humoral immune responses are also affected by allergen-SIT. Allergen-specific IgE levels transiently increase during the first months, but decline in the later course of allergen-SIT [27]. Circulating allergen-specific IgG antibodies show a gradual increase starting from the early phases of allergen-SIT. Allergen-specific IgG4 levels show the strongest increase during the course of allergen-SIT. This can result in decreases in the ratio of allergen-specific IgE:IgG4 of 10- to 100-fold $[28,29 \bullet \bullet]$. IgG antibodies can be directed against the same epitopes as IgE antibodies, and therefore could function as blocking antibodies that compete with IgE for the same epitope. There have been mixed reports on the correlation between increases in allergen-specific IgG levels and clinical outcome of allergen-SIT [30, 31]. It can be argued that, rather than the absolute concentration of allergen-specific IgG antibodies, one should assess the affinity and/or blocking activity of allergen-specific IgG to find a good correlation with clinical outcome of allergen-SIT. The decreased ratio of allergen-specific $\mathrm{IgE} / \mathrm{IgG} 4$ that is frequently observed during the course of allergen-SIT can be attributed to some extent to a skewing from allergen-specific Th2 cells towards Treg cell predominance. These cells could play a role in regulating both IgE and IgG4 production. Both IgG4 and IgE are induced upon stimulation with IL-4 and CD40L. The addition of IL-10, however, has opposite effects on IgE and IgG4 production. IgE production is suppressed by IL-10 while IgG4 production is augmented in the presence of IL-10 [32]. Furthermore, it has been shown that co-culturing IL-4+CD40L-stimulated peripheral blood mononuclear cells (PBMCs) as well as B cells with IL-10-secreting Tr1 cells or CD4+CD25+ Treg cells results in reduced IgE production and increased IgG4 production. In contrast, addition of CD4+CD25- effector T cells to IL-4+CD40L-stimulated PBMC or B cells did not affect IgE or IgG4 production [33]. These findings indicate that IL-10, Treg, and Tr1 cells regulate antibody isotype formation and skew the specific response from an IgE- to an IgG4-dominated phenotype. IgG4 is considered a non-inflammatory antibody isotype because of its low affinity for activating Fcy receptors and poor complement activating capacity. Furthermore, different IgG4 molecules can exchange the Fab arms, leading to the generation of bispecific, functionally monovalent antibodies that are unable to form immune complexes [34, 35].

- IL-10-producing regulatory B cells have been associated with protection against chronic inflammatory diseases, such as autoimmune disease and allergies [36]. Recently, we demonstrated that in bee venom allergic patients, $\mathrm{B}$ cells specific for the major bee venom al- 
lergen phospholipase A2 showed increased IL-10 production after bee venom SIT. Furthermore, we demonstrated that IL-10-producing regulatory B cells specifically upregulate production of non-inflam-

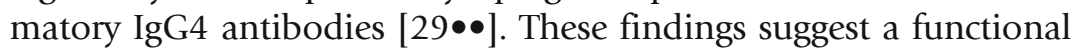
role for human regulatory B cells in allergen-SIT.

- There are several regimens for the application of allergen-SIT. The application routes used for allergen-SIT that are currently used in patient care are subcutaneous immunotherapy (SCIT) and, less frequently, sublingual immunotherapy (SLIT). Several other SIT approaches are currently being tested in clinical trials. There is moderate evidence for the efficacy of both SLIT and SCIT for the treatment of asthma $[37,38]$. SCIT may require more than 50 injections of allergen over a period of 3-5 years. This intensive trajectory results in poor patient compliance and high costs of treatment. Intralymphatic immunotherapy (ILIT) involves injection of allergens or allergen-constructs directly into the lymph node. Recent doubleblind, placebo-controlled trials investigated the efficacy and safety profile of ILIT for the treatment of pollen allergy and cat allergy. These initial studies demonstrated some improvement of allergic symptoms in response to three intralymphatic inguinal injections $[39 \bullet, 40]$. Therefore, ILIT may provide a more efficient method for the application of allergen-SIT. Other possible application routes for allergen-SIT that are currently being explored are epicutaneous and intradermal injection, as well as oral and intranasal application [41, $42 \bullet, 43]$.

- Modification of allergen preparations presents another opportunity for improving allergen-SIT. Allergens can be modified in order to prevent IgE binding, while retaining the capacity to target $\mathrm{T}$ cells. This prevents mast cell and basophil activation and may help to reduce the side effects of allergen-SIT. Allergen peptides, alternatively folded allergens, fragmented allergens or allergen polymers have reduced IgE but can retain the capacity to induce T cell responses [12]. Coupling of allergens to an adjuvant that triggers pattern recognition receptors is another exciting research area. Toll-like receptor (TLR) 4 and TLR9 agonists are highly immunogenic and are currently tested for their capacity enhance the efficacy of allergen-SIT. [44]

- One important issue regarding allergen-SIT for the treatment of allergic asthma is the fact that the majority of clinical trials have been designed to address the efficacy of allergen-SIT in patients with allergic rhinitis, and only a fraction of these patients (approximately $30 \%$ ) have rhinitis and asthma. Therefore, most of the published clinical trials are underpowered on asthma symptoms as a primary outcome. Furthermore, there is no consensus regarding the optimal endpoints to determine the effect of treatment on asthma. Therefore, the role of allergen-SIT in asthma has not been fully clarified so far.

- Adverse effects may occur as a result of allergen-SIT. Reported adverse effects of SCIT include local reactions (such as swelling) and systemic 
reactions (including anaphylaxis, asthma, rhinitis, and urticaria). The pooled relative risk for systemic reaction was 2.45 (95\% CI 1.913.13), while the pooled relative risk for local reactions was $1.4(95 \%$ CI 0.97-2.02) [37]. Reported adverse effects of SLIT include local reactions caused by grass pollen, tree pollen, house dust mite, and other allergens $(0.2-97 \%$ of SLIT-treated patients versus $3-38.5 \%$ of placebo-treated patients). Local reactions in the upper and lower respiratory tract as well as cutaneous reactions have been reported. Systemic reactions have not been reported in response to SLIT [38].

Emerging therapies

- A complex network of cytokine-mediated activation and suppression of tissue and immune cells underlies the development of asthma. Specific modulation of the major cytokines that act in this network is an important area of novel drug development for the treatment of asthma. The fact that certain cytokines are functionally redundant poses a challenge to the success of this approach. Currently, a number cytokine- and cytokine receptor-blocking antibodies are undergoing clinical testing. These include blocking antibodies against IL-2R $\alpha$, IL-4/IL-4R $\alpha$, IL-5, IL-9, IL-13/IL-13R $\alpha$, and TNF- $\alpha$. Additional cytokine-blocking antibodies [directed against IL-17, IL25, IL-31, IL-33, and thymic stromal lymphopoietin (TSLP)] are currently in pre-clinical development. As these drugs are still under development, we will focus primarily on the mechanism of action and outcomes of clinical trials that have been published so far.

- IL-2 is a key cytokine involved in proliferation and survival of activated effector T cells as well as $\mathrm{CD} 25^{+} \mathrm{FOXP}^{+}$regulatory (Treg) cells. Inhibition of IL-2R signaling interferes with $\mathrm{T}$ cell proliferation and suppresses cytokine production by activated effector $T$ helper $\left(\mathrm{T}_{\mathrm{H}}\right)$ cells. A humanized anti-IL-2R $\alpha$ monoclonal antibody (mAb) was evaluated in a randomized, double-blind, placebo-controlled study. The inclusion criteria were moderate to severe asthma patients who demonstrated reversible airflow obstruction despite inhaled corticosteroid treatment. Treated patients showed improved pulmonary function, significant reductions in asthma symptoms and the need for rescue medication, lower levels of blood eosinophilia, and longer exacerbation-free intervals. The majority $(65 \%)$ of the included patients (both in the treated and placebo groups) were atopic (based on sensitization towards at least three common allergens) [45].

- IL-4 and IL-13 are key mediators of the $\mathrm{T}_{\mathrm{H}} 2$ response as they induce $\mathrm{T}_{\mathrm{H}} 2$ differentiation of naïve CD4 ${ }^{+} \mathrm{T}$ cells and promote IL-4, IL-5, and IL-13 production by differentiated $\mathrm{T}_{\mathrm{H}} 2$ cells, as well as the induction of IgE class-switch recombination in B cells [46]. The effect of interfering with IL-4 signaling has been evaluated in several clinical trials using anti-IL-4-blocking mAbs as well as soluble IL-4R. These 
studies could not demonstrate any beneficial effect on asthma symptoms and exacerbations. An explanation for the failure of antiIL-4 treatment was sought in the functional redundancy between IL-4 and IL-13. Since both cytokines require IL-4R $\alpha$ for signaling, drugs targeting IL-4R $\alpha$ may be more effective. Indeed, treatment of atopic asthma patients with a mutated form of IL- 4 that blocks IL- $4 \mathrm{R} \alpha$ (pitrakinra, $1 \times 25 \mathrm{mg}$ subcutaneous daily or $60 \mathrm{mg}$ nebulized twice daily for 4 weeks) in a phase 2 randomized, double-blind, placebocontrolled trial improved lung function upon allergen challenge, and reduced the resting inflammatory status [47]. In another phase 2 trial using anti-IL-4R $\alpha$ mAbs (AMG-317, 6 weeks, $1 \times 75-300$ mg weekly) did not demonstrate clinical efficacy across the overall group of included patients based on the Asthma Control Questionnaire (ACQ), while a subgroup of patients with a high baseline ACQ score showed clinically significant improvements [48]. Several humanized mAbs against IL-13 are currently under development. The humanized antiIL-13 mAb lebrikizumab effectively improved lung function [as measured by forced expiratory volume in $\left.1 \mathrm{~s}\left(\mathrm{FEV}_{1}\right)\right]$ in patients with poorly controlled asthma despite glucocorticoid therapy. Patients with higher pretreatment levels of serum periostin, an IL-13-induced protein that plays a role in airway remodeling, showed the strongest improvement of lung function upon treatment, while patients with low periostin levels did not show significant improvement [49]. Therefore, serum periostin could be used as a biomarker to predict responsiveness to anti-IL-13 therapy. Therapies targeting IL-4 and IL13 are primarily aimed at severely allergic asthma patients.

- IL-5 is a key factor for eosinophil differentiation and survival and, because of the frequently observed eosinophilia in lung and circulation in asthma patients, it is a promising drug target for asthma treatment. Treatment of patients with persistent asthma with a humanized anti-IL-5 mAb (reslizumab) effectively reduced the levels of circulating eosinophils. There were, however, no observed effects on asthma symptoms, lung eosinophils, or $\mathrm{FEV}_{1}$ [50]. Another humanized anti-IL-5 mAb (mepolizumab) reduced serum (100\%) and lung (55\%) eosinophilia in patients with mild atopic asthma. Again, no significant effects on asthma symptoms were observed [51]. A later study highlighted the importance of stringent patient stratification for clinical trials for potential asthma drugs. Mepolizumab was tested in a randomized, double-blind, parallel-group trial on a rare subgroup of patients who continued to display sputum eosinophilia and airway symptoms despite continued treatment with prednisone. In this patient group a significant decrease in the number of sputum and blood eosinophils was observed, as well as a significant reduction in asthma exacerbations and improved $\mathrm{FEV}_{1}$ scores [52]. These findings suggest sputum eosinophilia could be used as a biomarker to predict responsiveness to anti-IL-5 therapy.

- TNF- $\alpha$ is a pro-inflammatory cytokine that plays a role in many aspects of the airway pathology in asthma, especially in refractory 


\section{Small molecules}

asthma. TNF- $\alpha$ mediates recruitment of neutrophils to the lungs, induction of glucocorticoid resistance, myocyte proliferation, and stimulation of fibroblast growth and maturation into myofibroblasts [53]. Clinical trials using the soluble TNF- $\alpha$ receptor fusion protein etanercept demonstrated improved lung function, airway hyperresponsiveness, and quality of life symptom scores upon treatment of patients with refractory asthma [54]. The anti-TNF- $\alpha$ $\mathrm{mAb}$ golimumab did not lead to improved lung function or incidence of severe asthma exacerbations of patients with severe and uncontrolled asthma. Severe side effects of anti-TNF- $\alpha$ therapy occurred in the form of serious infections and malignancy, which occurred more frequently in treated patients [55].

- Several other cytokines are currently being studied for their role in the pathology of asthma. Neutralizing therapies for IL-9, IL-17, IL-25, IL-31, IL-33, and TSLP that might be beneficial for distinct endotypes of asthma are currently under investigation [12].

- Like the anti-IgE mAb omalizumab, most of the cytokine-targeting immunomodulatory drugs that are currently under development for the treatment of asthma are mAbs. Provided that the required doses and frequencies of application are comparable, the costs associated with cytokine-inhibiting therapies will most likely be in the same range as anti-IgE therapy.

\section{Chemokine receptor antagonists}

- Chemokine receptors are G-protein-coupled receptors that play a pivotal role in the migration of leukocytes.

- Eosinophils, $\mathrm{T}_{\mathrm{H}} 2$ cells, basophils, and mast cells express CCR3 and their recruitment to the lungs is mediated by chemokines that can bind CCR3 such as eotaxin-1, -2, and -3, as well as RANTES (regulated on activation, normal $\mathrm{T}$ cell expressed and secreted) and monocyte chemotactic protein-4 (MCP-4) [56]. Therefore, interfering with CCR3 ligation has been proposed as a therapeutic strategy for the treatment of allergic asthma with eosinophilia. However, a recent double-blind parallel-group study of 60 asthma patients with $>4.9 \%$ sputum eosinophils did not show a significant reduction in sputum or blood eosinophil counts or improvement of lung function [57]. These observations challenge the role of CCR3 in airway eosinophilia in asthma.

- Neutrophil infiltration into the lungs is mediated by CXCR2. A clinical trial using the CXCR2 antagonist SCH527123 in patients with severe neutrophilic asthma demonstrated a reduction in the percentage of sputum neutrophils and the incidence of mild exacerbations. No serious adverse effects were reported [58]. CXCR2 antagonist therapy may benefit patients with severe neutrophilic asthma. 
- TLRs are key modulators of immune responses and function as sensors for danger signals such as microbial presence. Single SNPs (single nucleotide polymorphisms) and haplotypes of both TLR7 and 8 have been associated with increased risk of asthma [59]. TLR agonists are promising candidates for treatment and prevention of allergic asthma as they skew the balance of the allergic immune response from TH2 towards TH1. TLR4 and TLR9 agonists have been shown to improve allergen-SIT, as discussed above. Applications of TLR7 and TLR8 agonists (such as resiquimod and imiquimod) as standalone therapy have been tested in murine models. Skin application of imiquimod induced alterations of the respiratory leukocyte composition including an increase of DCs and natural killer cells, indicating that skin administration of this drug is a non-invasive means to modulate respiratory immunity [60]. Furthermore, the TLR7 agonist imiquimod and the TLR8 agonists (polyuridylic acid and polyadenylic acid) caused rapid dose-dependent relaxation of methacholine-contracted human airways in vitro through nitric oxide production [61]. The TLR7 agonist AZD8848 and the TLR8 agonist VTX-1463 have been tested in phase 2 clinical trials and reduced nasal symptoms in allergic rhinitis patients $[62,63]$. Recently, a novel TLR9 agonist (QbG10) showed clinical efficacy in persistent allergic asthma. These patients were subjected to controlled steroid withdrawal while treated with QbG10 or placebo. QbG10-treated patients reported better controlled asthma and better lung function than placebo-treated patients [64•].

- Thus, TLR agonists have the potential to function as adjuvant for allergen-SIT, to decrease the allergic Th2 response, and to act as immediate bronchodilators.

\section{Pediatric considerations}

- The approved immunomodulatory treatments that are currently used in clinical practice are anti-IgE therapy and allergen-SIT. Anti-IgE therapy with omalizumab is currently indicated for the treatment of moderate to severe asthma in patients older than 11 years of age [16]. A recent study provided supportive evidence for the safety and efficacy of omalizumab for the treatment of children aged 6-12 years with persistent allergic asthma [18•]. Allergen-SIT (in the form of SCIT) has been approved for the treatment of pediatric asthma. SLIT is not regulated but is used in clinical practice. There is moderate evidence that SCIT improves asthma symptoms in children and high evidence that SLIT improves asthma symptoms in children [65]. The observed adverse effects of both SCIT and SLIT consist primarily of 
local reactions. One SCIT trial reported anaphylaxis in one out of 16 HDM allergic patients [65].

\section{Compliance with Ethics Guidelines}

\section{Conflict of Interest}

Mübeccel Akdis and Willem van de Veen declare that they have no conflict of interest.

\section{Human and Animal Rights and Informed Consent}

This article does not contain any studies with human or animal subjects performed by any of the authors.

\section{References and Recommended Reading}

Papers of particular interest, published recently, have been

highlighted as:

- Of importance

• Of major importance

1. Bousquet J, Khaltaev N. Global surveillance, prevention and control of chronic respiratory diseases: a comprehensive approach. Global alliance against chronic respiratory diseases. Geneva: World Health Organization; 2007.

2. Agache I et al. Untangling asthma phenotypes and endotypes. Allergy. 2012;67(7):835-46.

3. Fitzpatrick AM et al. Heterogeneity of severe asthma in childhood: confirmation by cluster analysis of children in the National Institutes of Health/National Heart, Lung, and Blood Institute Severe Asthma Research Program. J Allergy Clin Immunol. 2011;127(2):382-9.

e1-13

4. Siroux V et al. Identifying adult asthma phenotypes using a clustering approach. Eur Respir J. 2011;38(2):310-7.

5. $\quad$ Lotvall J et al. Asthma endotypes: a new approach to classification of disease entities within the asthma syndrome. J Allergy Clin Immunol. 2011;127(2):355-60.

In this study the authors suggest new clasifications of asthma endotypes, which is proposed as a subtype of a condition defined by a distinct functional and pathophysiological mechanism. This new clasification aims to describe distinct disease entities with a defining molecular mechanisms, and is expected to facilitate better therapy that specifically targets the cause of the disease.

6. Anderson GP. Endotyping asthma: new insights into key pathogenic mechanisms in a complex, heterogeneous disease. Lancet. 2008;372(9643):1107-19.
7. Guy MC et al. Relationship between smokers' modes of entry into quitlines and treatment outcomes. Am J Health Behav. 2012;36(1):3-11.

8. Eder W, Ege MJ, von Mutius E. The asthma epidemic. N Engl J Med. 2006;355(21):2226-35.

9. Boulet LP. Asthma and obesity. Clin Exp Allergy. 2013;43(1):8-21.

10. Elazab $\mathrm{N}$ et al. Probiotic administration in early life, atopy, and asthma: a meta-analysis of clinical trials. Pediatrics. 2013;132(3):e666-76.

11. von Mutius E, Vercelli D. Farm living: effects on childhood asthma and allergy. Nat Rev Immunol. 2010;10(12):861-8.

12. Akdis CA. Therapies for allergic inflammation: refining strategies to induce tolerance. Nat Med. 2012;18(5):736-49.

13. Prussin $\mathrm{C}$ et al. Omalizumab treatment downregulates dendritic cell FcepsilonRI expression. J Allergy Clin Immunol. 2003;112(6):1147-54.

14. MacGlashan Jr DW et al. Down-regulation of $\mathrm{Fc}($ epsilon)RI expression on human basophils during in vivo treatment of atopic patients with anti-IgE antibody. J Immunol. 1997;158(3):1438-45.

15. Holgate ST et al. Anti-immunoglobulin E treatment with omalizumab in allergic diseases: an update on anti-inflammatory activity and clinical efficacy. Clin Exp Allergy. 2005;35(4):408-16.

16. Norman G et al. Omalizumab for the treatment of severe persistent allergic asthma: a systematic review and economic evaluation. Health Technol Assess. 2013;17(52):1-342. 
17. $\quad$ Busse W et al. High eosinophil count: a potential biomarker for assessing successful omalizumab treatment effects. J Allergy Clin Immunol.

2013;132(2):485-6e11.

This study demonstrates that eosinophil count may be used as a biomarker to predict omalizumab treatment outcomes, and should be further investigated. The patients who have high peripheral blood eosinophils may benefit from treatment with omalizumab.

18. Busse WW et al. Randomized trial of omalizumab (anti-IgE) for asthma in inner-city children. N Engl Med. 2011;364(11):1005-15.

This study is a randomized, double-blind, placebo-controlled, parallel-group trial at multiple centers to assess the effectiveness of omalizumab in inner-city children, adolescents, and young adults with persistent asthma.

Omalizumab improved asthma control, decreased seasonal peaks in exacerbations, and reduced the need for other medications to control asthma.

19. Di Domenico $M$ et al. Xolair in asthma therapy: an overview. Inflamm Allergy Drug Targets. 2011;10(1):2-12.

20. Noon L, Cantab BC. Prophylactic inoculation against hay fever. Lancet. 1911;1572-3.

21. Freeman J, Noon L. Further observation on the treatment of hay-fever by hypodermic inoculation of pollen vaccine. Lancet. 1911;2:814-7.

22. Burks AW et al. Update on allergy immunotherapy: American academy of allergy, asthma \& immunology/european academy of allergy and clinical immunology/PRACTALL consensus report. J Allergy Clin Immunol. 2013;131(5):128896.

e3

23. Novak N et al. Early suppression of basophil activation during allergen-specific immunotherapy by histamine receptor 2. J Allergy Clin Immunol. 2012;130(5):1153-1158.e2.

This study shows the role of HR2 on the early desensitization of basophils in bee venom allergic individuals during allergen-specific immunotherapy.

24. Akdis CA, Blaser K. Role of IL-10 in allergen-specific immunotherapy and normal response to allergens. Microbes Infect. 2001;3(11):891-8.

25. Akdis CA, Blaser K. IL-10-induced anergy in peripheral $\mathrm{T}$ cell and reactivation by microenvironmental cytokines: two key steps in specific immunotherapy. FASEB J. 1999;13(6):603-9.

26. Ling EM et al. Relation of CD4+CD25+ regulatory $\mathrm{T}$-cell suppression of allergen-driven T-cell activation to atopic status and expression of allergic disease. Lancet. 2004;363(9409):60815.

27. Akdis CA, Akdis M. Mechanisms of allergen-specific immunotherapy. J Allergy Clin Immunol. 2011;127(1):18-27.

quiz 28-9
28. Jutel $\mathrm{M}$ et al. Allergen-specific immunotherapy with recombinant grass pollen allergens. J Allergy Clin Immunol. 2005;116(3):608-13.

$29 . \bullet \quad$ van de Veen W et al. IgG4 production is confined to human IL-10-producing regulatory B cells that suppress antigen-specific immune responses. J Allergy Clin Immunol. 2013;131(4):1204-12.

In this article, role of $\mathrm{B}$ regulatory cells that produce IL-10 and later on switch to IgG4-producing plasma cells were shown for the first time. IL-10+ phospholipase A2 (PLA)specific $B$ cell frequency increased in allergic patients receiving PLA-specific immunotherapy.

30. Golden DB et al. Clinical relevance of the venomspecific immunoglobulin $G$ antibody level during immunotherapy. J Allergy Clin Immunol. 1982;69(6):489-93.

31. Muller U, Helbling A, Bischof M. Predictive value of venom-specific IgE, IgG and IgG subclass antibodies in patients on immunotherapy with honey bee venom. Allergy. 1989;44(6):412-8.

32. Jeannin $P$ et al. IgE versus IgG4 production can be differentially regulated by IL-10. J Immunol. 1998;160(7):3555-61.

33. Meiler F et al. Distinct regulation of IgE, IgG4 and IgA by $\mathrm{T}$ regulatory cells and toll-like receptors. Allergy. 2008;63(11):1455-63.

34. Aalberse RC et al. Immunoglobulin G4: an odd antibody. Clin Exp Allergy. 2009;39(4):469-77.

35. van der Neut Kolfschoten $\mathrm{M}$ et al. Anti-inflammatory activity of human IgG4 antibodies by dynamic Fab arm exchange. Science. 2007;317(5844):1554-7.

36. Mauri C, Bosma A. Immune regulatory function of B cells. Annu Rev Immunol. 2012;30:221-41.

37. Abramson MJ, Puy RM, Weiner JM. Injection allergen immunotherapy for asthma. Cochrane Database Syst Rev. 2010;8, CD001186.

38. Lin SY et al. Sublingual immunotherapy for the treatment of allergic rhinoconjunctivitis and asthma: a systematic review. JAMA. 2013;309(12):1278-88.

39. Hylander T et al. Intralymphatic allergen-specific immunotherapy: an effective and safe alternative treatment route for pollen-induced allergic rhinitis. J Allergy Clin Immunol. 2013;131(2):412-20.

In an open pilot investigation followed by a doubleblind,placebo-controlled study, patients with allergic rhinitis were treated with three intralymphatic inguinal injections of birch pollen or grass pollen or placebo. Intralymphatic immunotherapy with grass-pollen or birch-pollen extracts reduced nasal allergic symptoms without causing any safety problems.

40. Senti G et al. Intralymphatic immunotherapy for cat allergy induces tolerance after only 3 injections. J Allergy Clin Immunol. 2012;129(5):1290-6.

41. Rotiroti G et al. Repeated low-dose intradermal allergen injection suppresses allergen-induced cutaneous late responses. J Allergy Clin Immunol. 2012;130(4):918-24.e1. 
42. Senti G et al. Epicutaneous allergen-specific immunotherapy ameliorates grass pollen-induced rhinoconjunctivitis: a double-blind, placebo-controlled dose escalation study. J Allergy Clin Immunol. 2012;129(1):128-35.

This single-center, placebo-controlled, double-blind trial included patients with grass pollen-induced rhinoconjunctivitis. Patients were randomly selected to receive placebo or three different doses of allergen by using a patch to optimize treatment dose and to demonstrate the efficacy and safety of epicutaneous immunotherapy.

43. Cox L et al. Allergen immunotherapy: a practice parameter third update. J Allergy Clin Immunol. 2011;127(1 Suppl):S1-55.

44. Bezemer GF et al. Dual role of Toll-like receptors in asthma and chronic obstructive pulmonary disease. Pharmacol Rev. 2012;64(2):337-58.

45. Busse WW et al. Daclizumab improves asthma control in patients with moderate to severe persistent asthma: a randomized, controlled trial. Am J Respir Crit Care Med. 2008;178(10):1002-8.

46. Akdis $\mathrm{M}$ et al. Interleukins, from 1 to 37 , and interferon-gamma: receptors, functions, and roles in dise1-70 eases. J Allergy Clin Immunol. 2011;127(3):701-21.

47. Wenzel S et al. Effect of an interleukin-4 variant on late phase asthmatic response to allergen challenge in asthmatic patients: results of two phase 2 a studies. Lancet. 2007;370(9596):1422-31.

48. Corren J et al. A randomized, controlled, phase 2 study of AMG 317, an IL-4Ralpha antagonist, in patients with asthma. Am J Respir Crit Care Med. 2010;181(8):788-96.

49. Corren J et al. Lebrikizumab treatment in adults with asthma. N Engl J Med. 2011;365(12):1088-98.

50. Kips JC et al. Effect of SCH55700, a humanized antihuman interleukin-5 antibody, in severe persistent asthma: a pilot study. Am J Respir Crit Care Med. 2003;167(12):1655-9.

51. Flood-Page PT et al. Eosinophil's role remains uncertain as anti-interleukin-5 only partially depletes numbers in asthmatic airway. Am J Respir Crit Care Med. 2003;167(2):199-204

52. Nair P et al. Mepolizumab for prednisone-dependent asthma with sputum eosinophilia. N Engl J Med. 2009;360(10):985-93.

53. Berry $\mathrm{M}$ et al. TNF-alpha in asthma. Curr Opin Pharmacol. 2007;7(3):279-82.

54. Berry MA et al. Evidence of a role of tumor necrosis factor alpha in refractory asthma. $\mathrm{N}$ Engl J Med. 2006;354(7):697-708.

55. Wenzel SE et al. A randomized, double-blind, placebo-controlled study of tumor necrosis factor-alpha blockade in severe persistent asthma. Am J Respir Crit Care Med. 2009;179(7):549-58.

56. Willems LI, Ijzerman AP. Small molecule antagonists for chemokine CCR3 receptors. Med Res Rev. 2010;30(5):778-817.

57. Neighbour H, et al. Safety and efficacy of an oral CCR3 antagonist in patients with asthma and eosinophilic bronchitis: a randomized, placebo-controlled clinical trial. Clin Exp Allergy. Epub 2013 Nov 28

58. Nair P et al. Safety and efficacy of a CXCR2 antagonist in patients with severe asthma and sputum neutrophils: a randomized, placebo-controlled clinical trial. Clin Exp Allergy. 2012;42(7):1097-103.

59. Moller-Larsen $S$ et al. Association analysis identifies TLR7 and TLR8 as novel risk genes in asthma and related disorders. Thorax. 2008;63(12):1064-9.

60. Hackstein $\mathrm{H}$ et al. Skin TLR7 triggering promotes accumulation of respiratory dendritic cells and natural killer cells. PLoS One. 2012;7(8):e43320.

61. Drake MG et al. Toll-like receptor 7 rapidly relaxes human airways. Am J Respir Crit Care Med. 2013;188(6):664-72.

62. Greiff L et al. Repeated intranasal TLR7 stimulation reduces allergen responsiveness in allergic rhinitis. Respir Res. 2012;13:53.

63. Horak F. VTX-1463, a novel TLR8 agonist for the treatment of allergic rhinitis. Expert Opin Investig Drugs. 2011;20(7):981-6.

64. Beeh KM et al. The novel TLR-9 agonist QbG10 shows clinical efficacy in persistent allergic asthma. J Allergy Clin Immunol. 2013;131(3):866-74.

In this proof-of-concept parallel-group, double-blind, randomized trial, the authors demonstrated clinical efficacy, safety, and tolerability of the novel TLR9 agonist QbG10 in patients with mild-to-moderate persistent allergic asthma.

65. Kim JM et al. Allergen-specific immunotherapy for pediatric asthma and rhinoconjunctivitis: a systematic review. Pediatrics. 2013;131(6):1155-67.

66. Lommatzsch $\mathrm{M}$ et al. Against all odds: anti-IgE for intrinsic asthma? Thorax. 2014;69(1):94-6.

67. Compalati E, Braido F, Canonica GW. An update on allergen immunotherapy and asthma. Curr Opin Pulm Med. 2014;20(1):109-17.

68. Castro $\mathrm{M}$ et al. Reslizumab for poorly controlled, eosinophilic asthma: a randomized, placebo-controlled study. Am J Respir Crit Care Med. 2011;184(10):1125-32.

69. Busse WW et al. Safety profile, pharmacokinetics, and biologic activity of MEDI-563, an anti-IL-5 receptor alpha antibody, in a phase I study of subjects with mild asthma. J Allergy Clin Immunol. 2010;125(6):1237-1244.e2. 